\title{
The Development of Marked Collateral Circulation due to Inferior Vena Cava Filter Occlusion in a Patient with Chronic Thromboembolic Pulmonary Hypertension Complicated with Anti-phospholipid Syndrome
}

\author{
Hajime Kasai ${ }^{1}$, Nobuhiro Tanabe ${ }^{1,2}$, Ken Koshikawa $^{1}$, Yasutaka Hirasawa ${ }^{1}$, \\ Toshihiko Sugiura ${ }^{1}$, Seiichiro Sakao ${ }^{1}$ and Koichiro Tatsumi ${ }^{1}$
}

\begin{abstract}
A 30-year-old Japanese man was diagnosed with chronic thromboembolic pulmonary hypertension (CTEPH) with lupus anticoagulants (LAs) in 2003. He underwent pulmonary endarterectomy after the placement of an inferior vena cava filter (IVCF) in 2004, and treatment with warfarin was continued. In 2014, IVCF occlusion and marked collateral circulation were noted during an examination for transient dyspnea; however, his warfarin level was within the therapeutic range for $88.9 \%$ of the time from 2003 to 2014 . We herein report a rare case of CTEPH and LAs with IVCF occlusion; in such cases, intense treatment may be required.
\end{abstract}

Key words: chronic thromboembolic pulmonary hypertension, inferior vena cava filter, occlusion, antiphospholipid syndrome, lupus anticoagulants

(Intern Med 56: 931-936, 2017)

(DOI: 10.2169/internalmedicine.56.7599)

\section{Introduction}

In the clinical setting, inferior vena cava filters (IVCFs) are used to prevent pulmonary thromboembolism (PTE) from deep vein thrombosis (DVT) in the lower limbs and pelvis. However, the long-term use of IVCFs has been reported to induce various complications, such as perforation of the inferior vena cava (IVC) and the development of thrombi in the IVCF $(1,2)$. Chronic thromboembolic pulmonary hypertension (CTEPH) is a form of pulmonary hypertension that is caused by non-resolving thrombi in the pulmonary arteries and pulmonary vascular remodeling (3). In patients with CTEPH, an IVCF is placed prior to the performance of pulmonary endarterectomy (PEA), in order to prevent the recurrence of PTE from DVT during the perioperative period $(4,5)$. We herein present the case of a patient with CTEPH that was complicated by antiphospholipid syndrome (APS), who developed IVCF occlu- sion accompanied by significant hyperplasia of the collateral vessels.

\section{Case Report}

A 30-year-old Japanese man was diagnosed with CTEPH and primary APS in December 2002 after continuously testing positive for lupus anticoagulants (LAs). He underwent PEA after the placement of a permanent monoconical IVCF (Greenfield $^{\mathrm{TM}}$ Vena Cava Filter; Boston Scientific, Natick, MA, USA) in May 2003. His pulmonary hemodynamics and symptoms improved after PEA and anticoagulation therapy with warfarin was continued. He visited our hospital for blood tests, which included the evaluation of his prothrombin time (PT)-international normalized ratio (INR) and Ddimer level, every month. From 2003 to 2013, he was stable and had no symptoms indicating the deterioration of his respiratory status or the recurrence of PTE and DVT. Moreover, from 2003 to 2013, warfarin remained within the

${ }^{1}$ Department of Respirology, Graduate School of Medicine, Chiba University, Japan and ${ }^{2}$ Department of Advanced Medicine in Pulmonary Hypertension, Graduate School of Medicine, Chiba University, Japan

Received for publication April 18, 2016; Accepted for publication July 18, 2016

Correspondence to Dr. Hajime Kasai, daikasai6075@yahoo.co.jp 


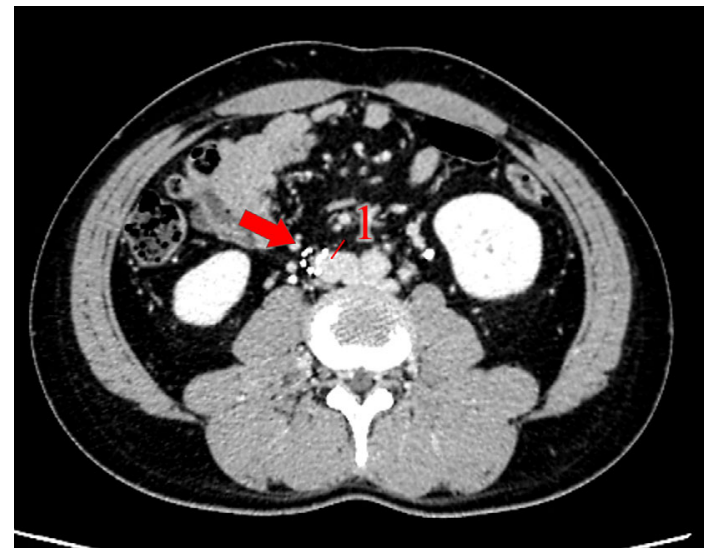

Figure 1. Contrast CT obtained in December 2013 showing an IVCF (arrow) that appeared to have been displaced outside the blood vessel. CT: computed tomography, IVCF: inferior vena cava filter. Arrow, IVCF; 1 , inferior vena cava

therapeutic range (PT-INR, 1.5-3.0) for $88.9 \%$ of the time; his D-dimer level remained at $<1.0 \mathrm{ng} / \mathrm{mL}$, and his platelet count was normal.

In October 2013, he experienced shortness of breath on exertion (modified Medical Research Council dyspnea scale grade 1). Contrast computed tomography (CT) did not reveal a recurrence of PTE; however, the IVCF appeared to have been displaced outside the blood vessel (Fig. 1). He was admitted to our department in January 2014 to evaluate the cause of his shortness of breath on exertion and to assess the complications of the indwelling IVCF. At the time of admission, the patient's shortness of breath on exertion had already disappeared. His body weight was $69 \mathrm{~kg}$ and his height was $168 \mathrm{~cm}$. His vital signs were as follows: blood pressure, $122 / 77 \mathrm{mmHg}$; pulse rate, 70 beats/min; $\mathrm{SpO}_{2}$ level while breathing room air, 95\%; and body temperature, $37.0^{\circ} \mathrm{C}$. He had no cardiac murmur and no abnormal respiratory sounds. He had no swelling or pain in his lower limbs.

Chest radiography, electrocardiography, and transthoracic echocardiography revealed no abnormalities. The patient's pulmonary function test results, including his $\mathrm{DL}_{\mathrm{Co}}$ level, proved to be normal as well. His D-dimer value was negative, and his PT-INR with the oral administration of warfarin (3.5 mg/day) was 1.79. His renal and liver function test results were normal. Repeat contrast CT (Aquilion One; Toshiba Medical, Tochigi, Japan) with a slice thickness of 2 $\mathrm{mm}, 0.35 \mathrm{~s} /$ rotation, and three-dimensional (3D) image reconstruction revealed the complete obstruction of the IVC at the site of the IVCF placement. The IVC showed aneurysmlike changes; blood flow below the occluded site of the IVC returned from the azygos vein to the superior vena cava via the collateral circulation (Fig. 2). There was no recurrence of CTEPH or obvious lung lesions. A retrospective review of follow-up contrast CT in 2006 revealed thrombi in the IVCF, but no DVT or collateral circulation were detected (Fig. 3).

Right heart catheterization revealed a normal mean pul- monary artery pressure of $18 \mathrm{mmHg}$, a pulmonary arterial wedge pressure of $7 \mathrm{mmHg}$, a pulmonary vascular resistance of 1.9 Wood units, and a cardiac index of $3.3 \mathrm{~L} / \mathrm{min} / \mathrm{m}^{2}$. Angiography of the IVC and the left iliac vein confirmed IVC occlusion at the site of the IVCF and the presence of significant collateral circulation, similar to that which was found by contrast CT (Fig. 4, 5). In addition, DVT was observed in the lower limb, and collateral circulation could not be detected. Pulmonary angiography revealed no evidence of acute or chronic PTE. Because he was asymptomatic at that time, we only offered close observation with strict anticoagulation therapy, with a target PT-INR of 2-3. For 3 years after discharge, until 2016, there were no signs indicating a recurrence of DVT or PTE.

\section{Discussion}

We presented a case with CTEPH that was complicated by APS in a patient who developed IVCF occlusion with significant hyperplasia of the collateral vessels. Thus far, there have been no reports about complications associated with indwelling IVCF in patients with CTEPH. Furthermore, we obtained detailed and interesting images of significant collateral circulation with the $3 \mathrm{D}$ reconstruction of contrast CT images.

The adaptation of an IVCF in PTE patients is controversial. Generally, an IVCF is inserted to prevent PTE in the perioperative period of PEA. All but one of the CTEPH patients who underwent IVCF placement at the time of PEA. The IVCF placement rate in the Japanese national registry of CTEPH patients was reported to be $26.9 \%$, while that in the international registry was $12.4 \%$ (6). Japanese patients were more likely to receive an IVCF, even in medically treated cases (6). Although IVCFs have been shown to reduce the recurrence of PTE, they do not have a significant effect on immediate or long-term mortality; moreover, they significantly increase the incidence of DVT $(7,8)$. It was recently reported that among hospitalized patients with severe acute PTE, the use of retrievable IVCFs plus anticoagulation therapy did not reduce the risk of symptomatic recurrent PTE in comparison to the administration of anticoagulation therapy alone (9). There are no reports concerning on the efficacy of IVCF in CTEPH patients; but the placement of an IVCF during the perioperative period, while anticoagulation therapy is stopped, has been performed at our institution because the University of California, San Diego, which is the largest center for the treatment of $\mathrm{CTPEH}$, routinely inserts IVCFs in the preoperative period.

Permanent IVCFs have been used in the past; however, retrievable IVCFs have been used because they can be removed once anticoagulant therapy is resumed after PEA. In fact, retrievable IVCFs should be removed when the initial indications no longer exist or when the contraindications to anticoagulation have resolved (10-12). Although the removal of retrievable IVCFs is recommended within 2 weeks after implantation (13), it is often not possible due to bleeding or 

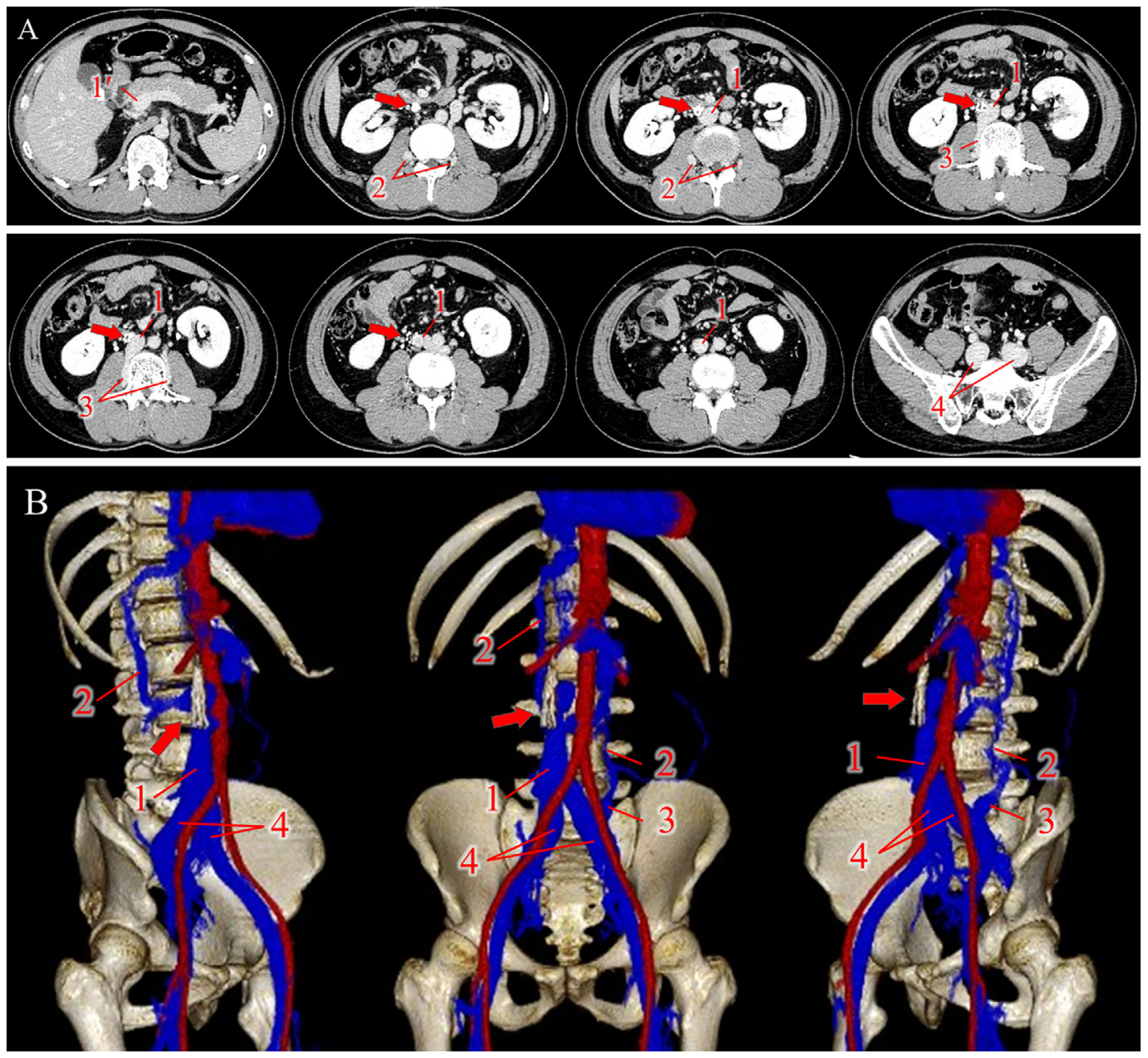

Figure 2. (A) A CT angiogram on admission showing a dilated and obstructed IVC (1) at the site of the indwelling IVCF (arrow). Blood flow below the site of IVC occlusion returned from the azygos vein to the superior vena cava via the collateral circulation $(2,3)$. In addition, both sides of the iliac vein (4) were markedly expanded. (B) A 3-dimensional image constructed from the contrast CT images showing the occlusion of the IVC and a detailed view of the collateral circulation. CT: computed tomography, IVC: inferior vena cava, IVCF: inferior vena cava filter. Arrow, IVCF; 1, IVC; 1', the remaining IVC from the renal vein of the head side; 2 , the ascending lumbar vein; 3 , the lumbar vein; 4, the iliac vein

prolonged intubation after PEA. In the present case, a permanent IVCF was inserted prior to PEA because longer postoperative intubation and immobilization were usual at that time. Meanwhile, the mean retrieval rate of retrievable IVCFs was $34 \%$, according to a recent systemic review of 37 studies (14) on PTE. Retrievable IVCFs are associated with significantly higher complication rates than permanent IVCFs $(15,16)$. The complications of retrievable IVCFs have been associated with their long-term use, and thus their early removal has been emphasized (14).

The incidence of IVCF occlusion is reported to be 2$30 \%$ (1). However, the incidence of IVCF occlusion in CTEPH patients has not been reported. In the present case, the thrombi in the IVCF on contrast CT were overlooked in 2006. The PT-INR values from 2003 to 2013 were as follows: 1.5 to $<2.0,54.3 \% ; 2.0$ to $<2.5,27.2 \%$; and 2.5 to $<$ $3.0,7.4 \%$. In addition, all of the D-dimer values were nega- tive during the same period, with the highest value being 0.7 $\mu \mathrm{g} / \mathrm{mL}$. With regard to coagulation abnormalities, the patient was only positive for LAs. Thus, in the present case, the predisposing condition for IVCF occlusion was presumed to be the thrombi in the patient's IVCF and APS. Xiao et al. (17) reported that the development of a thrombus in an IVCF reduced filter patency and venous return from the lower extremities and that it may progress to complete IVC occlusion. Hypercoagulable/malignant conditions may affect the formation of thrombi and IVCF occlusion (18). In addition, the biconical IVCF design also induces IVCF thrombosis because of the inverted conical design and the resultant flow dynamics (19). Reports have indicated that occlusion and/or the development of thrombi in the IVCF occurred in patients with polycythemia vera, heparin-induced thrombocytopenia, and patients without anticoagulants (20-22). Furthermore, there have been reports that occlusion and/or the 

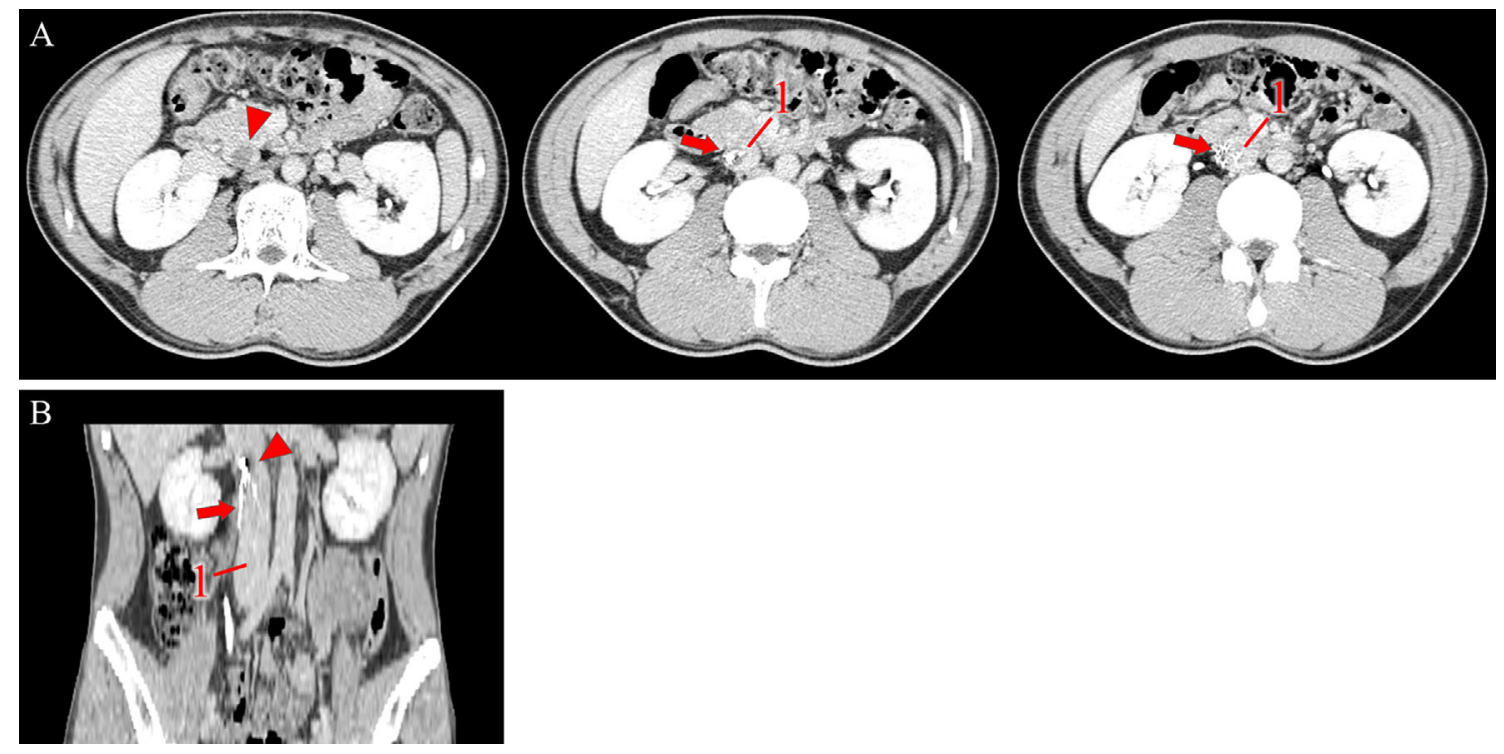

Figure 3. A retrospective review of follow-up contrast CT in 2006 showing thrombi (triangle) in the IVCF. CT: computed tomography, IVCF: inferior vena cava filter. Arrow, IVCF; 1, inferior vena cava

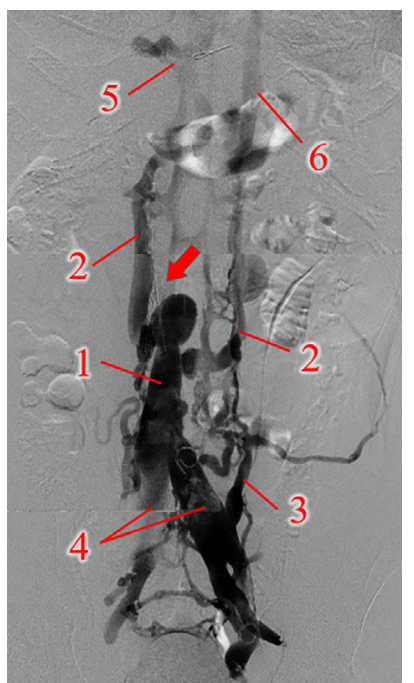

Figure 4. An angiogram of the IVC and left iliac vein confirming IVC occlusion at the site of the IVCF and the presence of marked collateral circulation, similar to the contrast CT findings. IVC: inferior vena cava, IVCF: inferior vena cava filter, CT: computed tomography (Arrow, IVCF; 1, IVC; 2, ascending lumbar vein; 3 , iliolumbar vein; 4 , iliac vein; 5 , azygos vein; 6 , hemi azygos vein)

development of a thrombus in the IVC occurred in a patient with hereditary protein $\mathrm{C}$ deficiency and APS, even without an indwelling IVCF (23-25). Thus, coagulation abnormalities may predispose the IVCF to occlusion. In particular, because LAs are found in $10-20 \%$ of patients with CTEPH $(26,27)$, it is necessary to pay more attention to IVCF occlusion in patients with CTEPH and other coagulation abnormalities.

The effectiveness of the concurrent use of anticoagulation therapy in patients in with an IVCF is controversial. Fur-

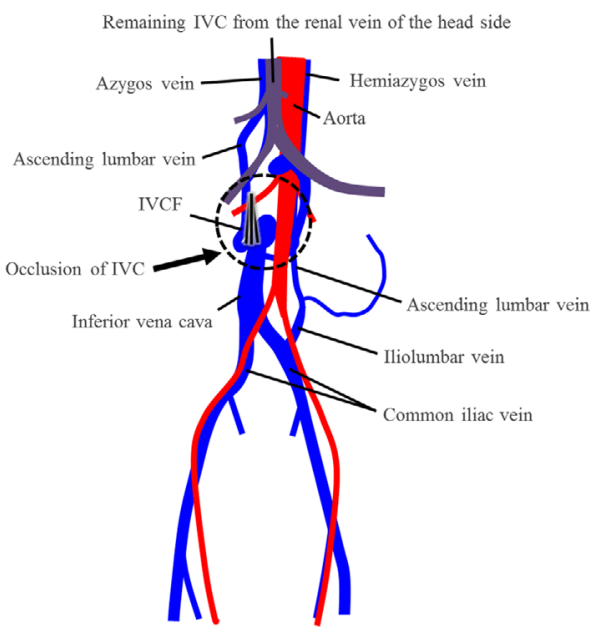

Figure 5. A schematic illustration of the 3-dimensional image constructed from the contrast $\mathrm{CT}$ images and angiography of the IVC. CT: computed tomography, IVC: inferior vena cava, IVCF: inferior vena cava filter

thermore, the optimal management of IVC thrombosis has not been established (21). Yazu et al. (28) reported that the incidence of IVC occlusion in Japanese patients with indwelling IVCFs significantly decreased after concurrent therapy. In Japan, anticoagulant therapy with a target PTINR in the range of 1.5-2.5, has been recommended for venous thromboembolism patients (29). On the other hand, patients with APS should be treated with a target PT-INR of 2.5 (target range, 2.0-3.0) (30). In our case, a PT-INR of $>2$ was only observed $34.6 \%$ of the time and was mostly controlled at a PT-INR of 1.5-2 from 2003 to 2013. For patients with accompanying APS, the use of our target PT-INR based on the Japanese guidelines, may not be sufficient. In contrast, Ahmad et al. (31) reported that there was no difference 
in the regression of thrombi in the IVCFs of a group of patients who received anticoagulation therapy and a group who did not. Thus, invasive treatments, such as percutaneous mechanical thrombectomy, stenting, and catheter-directed thrombolysis, should be considered after the detection of a thrombus in an IVCF $(17,21,22,32)$.

In the present case, IVCF occlusion was considered to have gradually developed because hyperplasia of the collateral circulation developed without swelling or pain in the lower limbs. Because of collateral vein drainage, some patients with IVCF thrombosis may not manifest lower extremity symptoms (17). Conversely, Gurewich et al. (33) reported that the resultant venous tributaries serve as alternative routes for PTE. The transient shortness of breath on exertion in the present case may have been caused by a recurrence of PTE through collateral circulation. Careful observation is therefore necessary. For example, the performance of contrast CT may even be useful in cases in which there is only a slight increase in the D-dimer and additional soluble fibrin monomer complex (SFMC) levels, which are markers of acute thrombosis. If possible, the IVCF should be removed after PEA, even in patients with CTEPH. In the present case, the thrombi in the IVCF were overlooked on contrast CT in 2006; the doctors may have mistaken the patient's thrombi for artifacts related to the IVCF at that time. In addition, this case demonstrated that 3D contrast CT images may be useful for the early detection of IVCF occlusion and the presence of collateral circulation.

We presented a case of CTEPH that was complicated with APS in a patient who developed IVCF occlusion that was accompanied by significant hyperplasia of the collateral vessels. In such cases, careful prolonged observation and more intense treatment may be required.

\section{Author's disclosure of potential Conflicts of Interest (COI).}

Nobuhiro Tanabe: Honoraria, Pfizer; Others, Actelion Pharmaceuticals. Koichiro Tatsumi: Honoraria, GlaxoSmithKline and Pfizer.

\section{Financial Support}

Koichiro Tatsumi received a grant from the Respiratory Failure Research Group from the Ministry of Health, Labor, and Welfare, Japan (No 21590962). Nobuhiro Tanabe received a research grant from the Ministry of Education, Culture, Sports, Science, and Technology of Japan (No 22590849), and received a grant from the Japanese Respiratory Society.

\section{References}

1. Caplin DM, Nikolic B, Kalva SP, et al. Quality improvement guidelines for the performance of inferior vena cava filter placement for the prevention of pulmonary embolism. J Vasc Interv Radiol: JVIR 22: 1499-1506, 2011.

2. Sella DM, Oldenburg WA. Complications of inferior vena cava filters. Semin Vasc Surg 26: 23-28, 2013.

3. Haythe J. Chronic thromboembolic pulmonary hypertension: a review of current practice. Progress in cardiovascular diseases 55: 134-143, 2012.
4. Coronel ML, Chamorro N, Blanco I, et al. Medical and surgical management for chronic thromboembolic pulmonary hypertension: a single center experience. Arch Bronconeumol 50: 521-527, 2014.

5. Jamieson SW, Kapelanski DP. Pulmonary endarterectomy. Curr Probl Surg 37: 165-252, 2000.

6. Tanabe N, Sugiura T, Tatsumi K. Recent progress in the diagnosis and management of chronic thromboembolic pulmonary hypertension. Respir Investig 51: 134-146, 2013.

7. Decousus H, Leizorovicz A, Parent F, et al. A clinical trial of vena caval filters in the prevention of pulmonary embolism in patients with proximal deep-vein thrombosis. Prevention du Risque d'Embolie Pulmonaire par Interruption Cave Study Group. N Engl J Med 338: 409-415, 1998.

8. Group PS. Eight-year follow-up of patients with permanent vena cava filters in the prevention of pulmonary embolism: the PREPIC (Prevention du Risque d'Embolie Pulmonaire par Interruption Cave) randomized study. Circulation 112: 416-422, 2005.

9. Mismetti P, Laporte S, Pellerin O, et al. Effect of a retrievable inferior vena cava filter plus anticoagulation vs anticoagulation alone on risk of recurrent pulmonary embolism: a randomized clinical trial. JAMA 313: 1627-1635, 2015.

10. Jaff MR, McMurtry MS, Archer SL, et al. Management of massive and submassive pulmonary embolism, iliofemoral deep vein thrombosis, and chronic thromboembolic pulmonary hypertension: a scientific statement from the American Heart Association. Circulation 123: 1788-1830, 2011.

11. Administration USFaD. Removing retrievable inferior vena cava filters: initial communication. http://www.fda.gov/MedicalDevices/ Safety/AlertsandNotices/ucm221676.htm

12. Kearon C, Akl EA, Comerota AJ, et al. Antithrombotic therapy for VTE disease: Antithrombotic Therapy and Prevention of Thrombosis, 9th ed: American College of Chest Physicians Evidence-Based Clinical Practice Guidelines. Chest 141: e419S-494S, 2012.

13. Torbicki A, Perrier A, Konstantinides S, et al. Guidelines on the diagnosis and management of acute pulmonary embolism: the Task Force for the Diagnosis and Management of Acute Pulmonary Embolism of the European Society of Cardiology (ESC). Eur Heart J 29: 2276-2315, 2008.

14. Angel LF, Tapson V, Galgon RE, Restrepo MI, Kaufman J. Systematic review of the use of retrievable inferior vena cava filters. $J$ Vasc Interv Radiol 22: 1522-1530 e1523, 2011.

15. Desai TR, Morcos OC, Lind BB, et al. Complications of indwelling retrievable versus permanent inferior vena cava filters. J Vasc Surg Venous Lymphat Disord 2: 166-173, 2014.

16. Andreoli JM, Lewandowski RJ, Vogelzang RL, Ryu RK. Comparison of complication rates associated with permanent and retrievable inferior vena cava filters: a review of the MAUDE database. $\mathrm{J}$ Vasc Interv Radiol 25: 1181-1185, 2014.

17. Xiao L, Shen J, Tong JJ, et al. Transcatheter thrombolytic therapy for symptomatic thrombo-occlusion of inferior vena cava filter. Exp Ther Med 5: 533-538, 2013.

18. Nazzal M, Chan E, Nazzal M, et al. Complications related to inferior vena cava filters: a single-center experience. Ann Vasc Surg 24: 480-486, 2010.

19. Corriere MA, Sauve KJ, Ayerdi J, et al. Vena cava filters and inferior vena cava thrombosis. J Vasc Surg 45: 789-794, 2007.

20. Muranishi H, Nakashima D, Nagata $H$, et al. Thrombo-occlusion of inferior vena cava filter in a patient with polycythemia vera. $\mathrm{J}$ Cardiol 54: 307-310, 2009.

21. Yan BP, Kiernan TJ, Gupta V, Ajani AE, Schainfeld RM. Combined pharmacomechanical thrombectomy for acute inferior vena cava filter thrombosis. Cardiovasc Revascu Med 9: 36-40, 2008.

22. Alkhouli M, Shafi I, Bashir R. Inferior vena cava filter thrombosis and suprarenal caval stenosis: a double whammy. JACC Cardiovasc Intervs 8: e23-e25, 2015.

23. Dhiman RK, Saraswat VA, Radhakrishnan S, Parashar A, Agarwal 
DK, Naik SR. Multiple venous thromboses and membranous obstruction of inferior vena cava in association with hereditary protein C deficiency: a case report. J Gastroenterol Hepatol 7: 434438, 1992.

24. Chacek S, MacGregor-Gooch J, Halabe-Cherem J, Nellen-Hummel $\mathrm{H}$, Quinones-Galvan A. Pyoderma gangrenosum and extensive caval thrombosis associated with the antiphospholipid syndrome--a case report. Angiology 49: 157-160, 1998.

25. Mukai Y, Tsutsui H, Todaka K, et al. Total occlusion of inferior vena cava in a patient with antiphospholipid antibody syndrome associated with behcet's disease. Jpn Circ J 65: 837-838, 2001.

26. Auger WR, Permpikul P, Moser KM. Lupus anticoagulant, heparin use, and thrombocytopenia in patients with chronic thromboembolic pulmonary hypertension: a preliminary report. Am J Med 99: 392-396, 1995.

27. Wolf M, Boyer-Neumann C, Parent F, et al. Thrombotic risk factors in pulmonary hypertension. Eur Respir J 15: 395-399, 2000.

28. Yazu T, Fujioka H, Nakamura M, et al. Long-term results of inferior vena cava filters: experiences in a Japanese population. Intern Med 39: 707-714, 2000.

29. Group JCSJW. Guidelines for the diagnosis, treatment and preven- tion of pulmonary thromboembolism and deep vein thrombosis (JCS 2009). Circ J 75: 1258-1281, 2011.

30. Keeling D, Mackie I, Moore GW, Greer IA, Greaves M; British Committee for Standards in H. Guidelines on the investigation and management of antiphospholipid syndrome. Br J Haematol 157: 47-58, 2012.

31. Ahmad I, Yeddula K, Wicky S, Kalva SP. Clinical sequelae of thrombus in an inferior vena cava filter. Cardiovasc Intervent Radiol 33: 285-289, 2010.

32. Neglen P, Oglesbee M, Olivier J, Raju S. Stenting of chronically obstructed inferior vena cava filters. J Vasc Surg 54: 153-161, 2011.

33. Gurewich V, Thomas DP, Rabinov KR. Pulmonary embolism after ligation of the inferior vena cava. New Engl J Med 274: 1350$1354,1966$.

The Internal Medicine is an Open Access article distributed under the Creative Commons Attribution-NonCommercial-NoDerivatives 4.0 International License. To view the details of this license, please visit (https://creativecommons.org/licenses/ by-nc-nd/4.0/).

(C) 2017 The Japanese Society of Internal Medicine http://www.naika.or.jp/imonline/index.html 